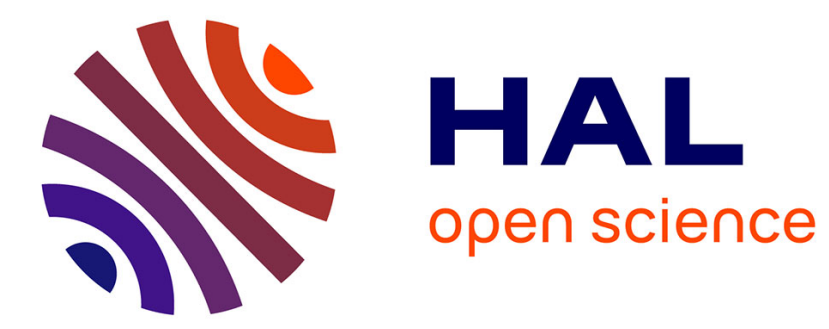

\title{
Filamentation of light in carbon disulfide
}

\author{
Valentin Besse, Hervé Leblond, Georges Boudebs
}

\section{To cite this version:}

Valentin Besse, Hervé Leblond, Georges Boudebs. Filamentation of light in carbon disulfide. Physical Review A : Atomic, molecular, and optical physics [1990-2015], 2014, 89 (4), pp.043840. 10.1103/PhysRevA.89.043840 . hal-03204330

\section{HAL Id: hal-03204330 \\ https://univ-angers.hal.science/hal-03204330}

Submitted on 21 Apr 2021

HAL is a multi-disciplinary open access archive for the deposit and dissemination of scientific research documents, whether they are published or not. The documents may come from teaching and research institutions in France or abroad, or from public or private research centers.
L'archive ouverte pluridisciplinaire HAL, est destinée au dépôt et à la diffusion de documents scientifiques de niveau recherche, publiés ou non, émanant des établissements d'enseignement et de recherche français ou étrangers, des laboratoires publics ou privés. 


\title{
Filamentation of light in carbon disulfide
}

\author{
V. Besse, H. Leblond, and G. Boudebs \\ LUNAM Université, Université d'Angers, Laboratoire de Photonique d'Angers, EA 4464, 2 Boulevard Lavoisier, 49000 Angers, France
}

(Received 29 January 2014; published 25 April 2014)

\begin{abstract}
We report experimental observation of light filamentation in carbon disulfide $\left(\mathrm{CS}_{2}\right)$. Accurate measurements of the nonlinear index show an unusual saturation law of the Kerr effect, which is used to build a model of light propagation in $\mathrm{CS}_{2}$, which describes the filamentation in good agreement with experimental observations.
\end{abstract}

DOI: 10.1103/PhysRevA.89.043840

PACS number(s): 42.65.Tg, 05.45.Yv, 42.70.Nq

\section{INTRODUCTION}

Filamentation of a high-intensity optical pulse was observed even before the concept of an optical soliton was introduced and first modeled through a purely cubic Kerr effect [1]. The high intensities reached in filaments allow one to observe many nonlinear phenomena such as highharmonic or supercontinuum generation (see the review in [2]). Permanent index changes induced by light filaments in glass were observed very early [3] and more recently used to burn waveguides (see, e.g., [4]). Filamentation in air has been especially studied; it was shown that the arrest of collapse is due to ionization [2], including multiple filamentation for powers in the terawatt range [5]. Whatever the reason for arresting collapse, many explanations have been proposed for the formation of the filament pattern such as polarization effects [6] and ellipticity of the beam [7]. Exotic beam shapes, such as Bessel beams [8] or unstable optical vortices [9], have been shown to lead to filamentation.

Recently, two-dimensional spatial soliton propagation essentially stabilized due to the $\chi^{(3)}-\chi^{(5)}$ balance in carbon disulfide $\left(\mathrm{CS}_{2}\right)$ at $\lambda=920 \mathrm{~nm}$ was reported [10] and we consider here light filamentation in this liquid. Carbon disulfide has been used for a long time as a reference material for nonlinear index measurements. Hence many experiments of nonlinear index characterization are available in the literature [10-15]. It is indeed commonly stated that $\mathrm{CS}_{2}$ possesses a third-order nonlinear refractive index $n_{2}$ that essentially does not vary with input intensity, at least if it remains moderate. We found that this statement is not true and even that it is not possible to correct it by merely adding a fifth-order index term $n_{4}$. Maybe it is the actual reason why very few papers, if any, report measurements of $n_{4}$ in $\mathrm{CS}_{2}$ at $\lambda=532 \mathrm{~nm}$ in the picosecond regime. Consequently, the standard model for the description of the nonlinear dynamics of a light beam in this medium, which is based on the cubic nonlinear Schrödinger (NLS) equation, eventually refined by adding several terms to account for the higher-order nonlinear index and nonlinear absorption, does not remain valid as an accurate description of the saturation of the Kerr effect is required, which is the case in modeling filamentation. We propose in this paper an alternative model based on accurate measurements of effective $n_{2}$.

\section{OBSERVATION OF THE FILAMENTATION}

First we investigate the propagation of a laser beam in a cell filled with $\mathrm{CS}_{2}$, with thickness $L=10 \mathrm{~mm}$. The experimental setup is shown in Fig. 1. The lens $\mathrm{L}_{1}\left(f_{1}=200 \mathrm{~mm}\right)$ is used to focus the light at the entry of the cell while the dashed box ( $\mathrm{L}_{2}$ and the CCD sensor) is an imaging system that can be translated following the $z$ axis to image the entry and the output of the cell (from $z=0$ to $z=L$, respectively). The ratio between $i$ and $o$ corresponds to a magnification equal to 10 . The light source is a Nd:YAG laser, operating at wavelength $\lambda=532 \mathrm{~nm}$ and emitting 12-ps-long pulses with Gaussian time profile, linearly polarized, operating at a repetition rate of $10 \mathrm{~Hz}$, which prevents any thermal effect. The output beam is acquired using a $(1024 \times 768)$-pixel $\left(6.45 \times 6.45 \mu \mathrm{m}^{2}\right.$ each $)$ CCD sensor triggered with the laser. At the entrance of the setup, a waveplate and a Glan prism allow one to control the input light intensity and to maintain linear polarization. Beam shaping is achieved using a spatial filter, so the spatial profile of the collimated beam is close to Gaussian at the input of the setup (see Fig. 1).

We set the Rayleigh distance to be $z_{R}=L / 10=1 \mathrm{~mm}$ by adjusting the beam waist in the focus of $\mathrm{L}_{1}$ to be $w_{0}=$ $\sqrt{z_{R} \lambda / \pi}=13 \mu \mathrm{m}$. Above an input energy per pulse of $1.4 \mu \mathrm{J}$, we observe the formation of filaments at the output of the cell (see Fig. 2). Changing the input intensity allows us to control the number of filaments.

\section{NONLINEAR INDEX MEASUREMENTS}

To give a theoretical account of these observations, an accurate measurement of the nonlinear parameters of $\mathrm{CS}_{2}$ is required. We perform it using the well-known $Z$-scan method [11]. The standard model for the evolution of the light intensity $I$ and the beam phase $\varphi$ during propagation in a nonlinear medium, in the thin sample approximation, i.e., if diffraction is negligible, can be set as

$$
\begin{gathered}
\frac{d I}{d z}=-\alpha I-\beta I^{2}-\gamma I^{3}, \\
\frac{d \varphi}{d z}=k n_{2, \mathrm{eff}}(I)=k\left(n_{2} I+n_{4} I^{2}\right),
\end{gathered}
$$

where $z$ is the spatial variable in the propagation direction; $k$ is the wave vector; $\alpha, \beta$, and $\gamma$ are the linear, two-photon, and three-photon absorption coefficients, respectively; and $n_{2}$ and $n_{4}$ are the third-order and fifth-order nonlinear refraction indices, respectively. Measurements using a nonlinear fitting of the curve yielded by numerical solution of the differential evolution equation and analytical solutions of Eqs. (1) and (2), with the necessary approximations, have been given $[16,17]$. Equations (1) and (2) can be solved analytically in special 


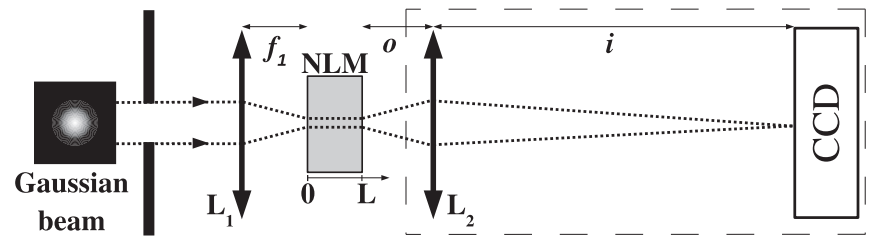

FIG. 1. Experimental setup used to produce filamentation: NLM, nonlinear medium $\left(\mathrm{CS}_{2}\right) ; \mathrm{L}_{1}$ and $\mathrm{L}_{2}$, lenses; $f_{1}$, focal length of $\mathrm{L}_{1}$; $\mathrm{CCD}$, digital camera; $i$, image distance; and $o$, object distance.

cases where some of the nonlinear coefficients vanish [18]. In the general case, an analytical expression of $z$ versus $I$ is found and inverted numerically [15].

It is known that $\mathrm{CS}_{2}$ has neither linear nor two-photon absorption $(\alpha=0, \beta=0)$. However, Kong et al. have reported an appreciable three-photon absorption $\gamma$ at $800 \mathrm{~nm}$ [19]; they also found the value of $\gamma=1.37 \times 10^{-27} \mathrm{~m}^{3} / \mathrm{W}^{2}$. Moreover, one of the absorption bands of $\mathrm{CS}_{2}$ is centered at about

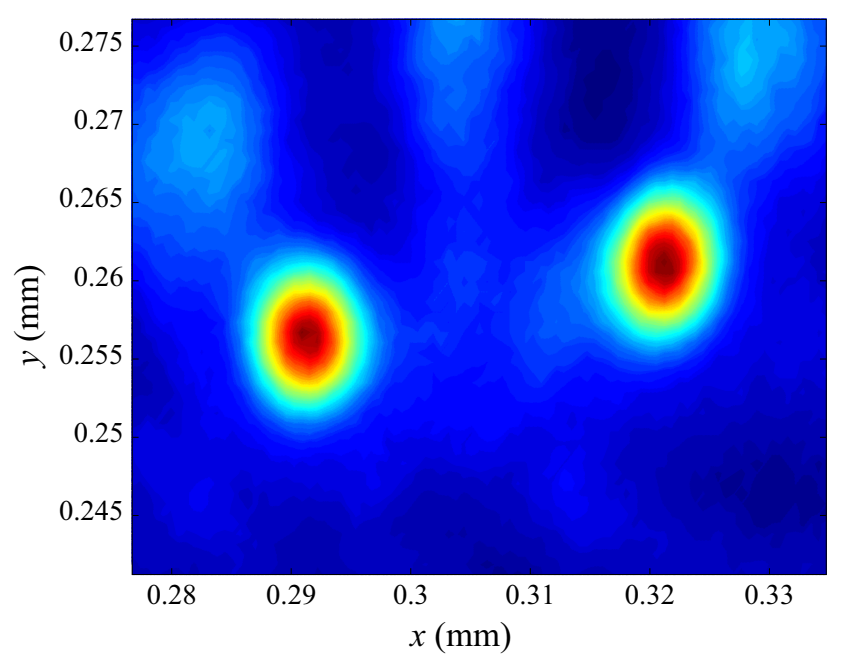

(a)

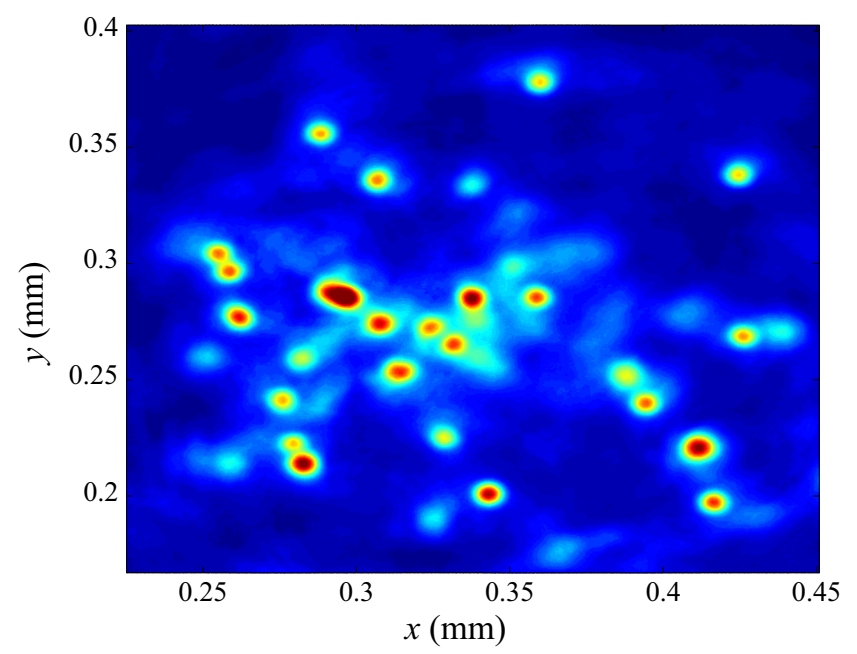

(b)

FIG. 2. (Color online) Filamentation observed at the output of the $\mathrm{CS}_{2}$ cell for different values of the pulse energy: (a) $2.7 \mu \mathrm{J}$ and (b) $14.2 \mu \mathrm{J}$.

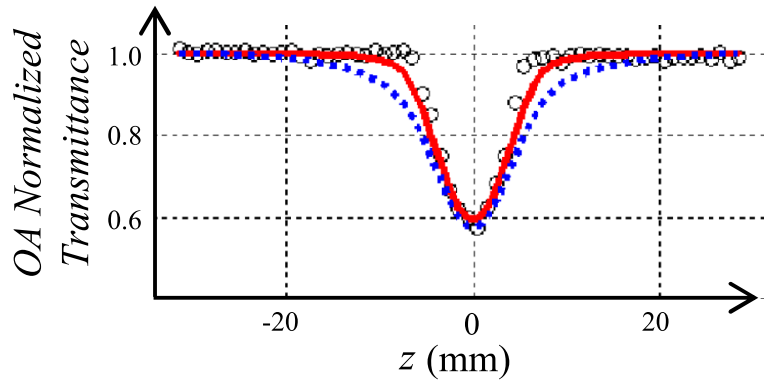

FIG. 3. (Color online) Normalized transmittance (open circles) obtained by the OA $Z$-scan with a 1 -mm-thick cell filled with $\mathrm{CS}_{2}$, measured at $\lambda=532 \mathrm{~nm}$ and $I_{0}=25 \mathrm{GW} / \mathrm{cm}^{2}$. The dashed (blue) line is a fitting using two-photon absorption [Eq. (3)] only, $\beta=$ $8.5 \times 10^{-12} \mathrm{~m} / \mathrm{W}$, while the solid (red) line shows the numerical fit considering three-photon absorption [Eq. (4)] only, $\gamma=9.3 \times$ $10^{-26} \mathrm{~m}^{3} / \mathrm{W}^{2}$. The radius of the circular object is $1.7 \mathrm{~mm}$, the beam diameter in the focal plane is $19 \mu \mathrm{m}$, and the focal length of lens $\mathrm{L}_{3}$ is $f_{3}=20 \mathrm{~cm}$.

$200 \mathrm{~nm}$ [10], which is approximately the third harmonic $\lambda / 3$ of the wavelength $\lambda=532 \mathrm{~nm}$. This closeness to resonance increases the fifth-order optical susceptibility $\left|\chi^{(5)}\right| \propto \gamma$ in this experiment.

We use the standard open aperture (OA) Z-scan method, together with analytic solution of (1) and (2) and numerical inversion of it [15]. The open circles in Fig. 3 corresponding to the experimental data are fitted with both Ansätze

$$
\begin{aligned}
& z=\frac{I_{0}-I_{L}}{\beta I_{0} I_{L}}, \\
& z=\frac{I_{0}^{2}-I_{L}^{2}}{2 \gamma I_{0}^{2} I_{L}^{2}},
\end{aligned}
$$

where $I_{0}$ is the input intensity at the entrance of the nonlinear medium, $I_{L}$ is the output one, and $L$ is the sample thickness. The best fit is obtained with the three-photon absorption, which confirms that $\mathrm{CS}_{2}$ does not exhibit two-photon absorption and gives the value of the three-photon absorption coefficient $\gamma=9.3 \times 10^{-26} \mathrm{~m}^{3} / \mathrm{W}^{2}$. The change in transmittance of the material is observed via a cooled CCD camera $\left(-30^{\circ} \mathrm{C}\right)$ with a sensor of $1000 \times 1018$ pixels $\left(12 \times 12 \mu \mathrm{m}^{2}\right)$.

The standard closed-aperture (CA) $Z$-scan [11] requires that the incident intensity is calibrated using a reference nonlinear medium (which is usually $\mathrm{CS}_{2}$ ). Recently, however, "absolute" measurement methods have emerged [20], avoiding the calibration. For this, the used setup is a $4 f$ system (see Fig. 4), consisting of two lenses $\mathrm{L}_{3}$ and $\mathrm{L}_{4}$. The top-hat beam object is located in the front focal plane focus of the lens $\mathrm{L}_{3}$ and its Fourier transform is obtained in the rear focal plane of the same lens, where the sample is placed, here a 1-mm-thick cell filled with $\mathrm{CS}_{2}$. The same point is also the front focal point of $\mathrm{L}_{4}$, which performs the Fourier transform into the camera. A recent improvement of the technique allows us to avoid the division of the two normalized $Z$-scan transmittances (closed and open aperture ones) by recording the beam waist relative variations according to the second-order momentum (the D4 $\sigma$ method) [21]. We measure the effective third-order refraction index $n_{2, \text { eff }}$ using the D $4 \sigma$ method, with increasing 


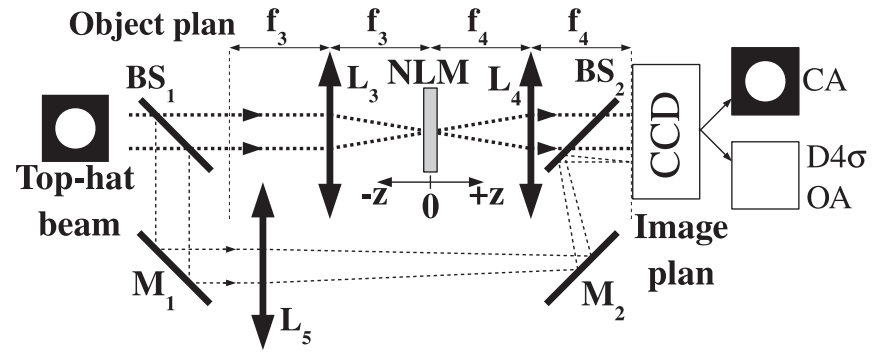

FIG. 4. Setup of the $Z$-scan measurement: BS, beam splitter; M, mirrors; $\mathrm{L}_{3}$ to $\mathrm{L}_{5}$, lenses with focal length $f_{3}$ to $f_{5}$; NLM, nonlinear medium; and CCD, digital camera. Using digital filters allows us to switch from one measurement method to another (D4 $\sigma$, OA, or CA).

intensity, for two wavelengths $\lambda=532$ and $1064 \mathrm{~nm}$ (see Fig. 5). We observe a steep increase of $n_{2}$ as the input intensity $I_{0}$ increases. Then, after having reached a maximum value, $n_{2}$ decreases rapidly again. We observe the same evolution for both wavelengths, except that, at $\lambda=1064 \mathrm{~nm}$, the variations are slower and the intensity range is wider.

These results call into question the use of $\mathrm{CS}_{2}$ as a reference material for calibrating nonlinear measurement systems. It is known that the response of materials may vary depending on the pulse duration, wavelength, and polarization. The nonlinear index coefficient $n_{2}$ cannot be defined unambiguously anymore since it is not a constant even in the weakly nonlinear regime. The definition of $n_{4}$ is a fortiori problematic.

\section{AN ADAPTED MODEL}

The measurements show that the usual formula $n_{2, \text { eff }}(I)=$ $n_{2}+n_{4} I$ cannot be used anymore. The situation differs greatly from the experiments of [10] and theory of [22] because the nonlinear index $n_{2 \text {,eff }}$ is here increasing with moderate intensities, instead of decreasing. In other words, $n_{4}$ is positive at the considered wavelengths and not negative as in [10]. Indeed, measurements performed at moderate intensities have provided the values $n_{4}=(1.2 \pm 0.3) \times 10^{-32} \mathrm{~m}^{4} / \mathrm{W}^{2}$ at $\lambda=532 \mathrm{~nm}$ and $n_{4}=(2.2 \pm 0.4) \times 10^{-33} \mathrm{~m}^{4} / \mathrm{W}^{2}$ at $\lambda=$

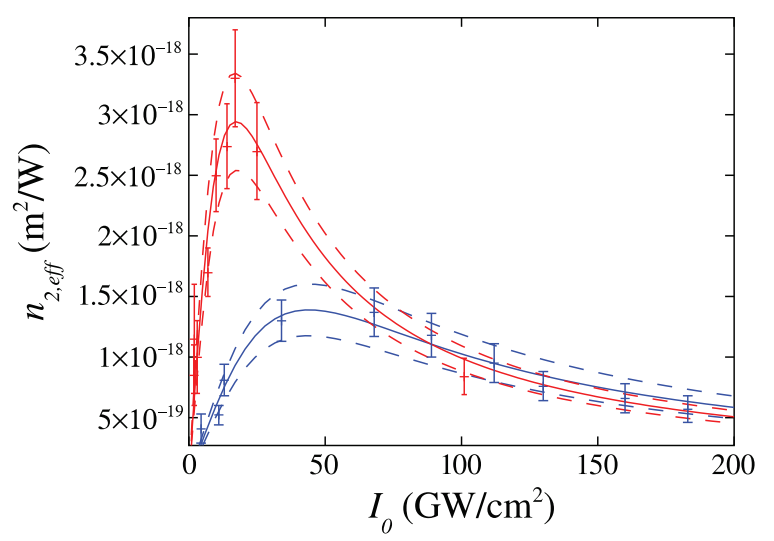

FIG. 5. (Color online) Plot of $n_{2, \text { eff }}\left(\mathrm{m}^{2} / \mathrm{W}\right)$ versus $I_{0}\left(\mathrm{~W} / \mathrm{m}^{2}\right)$ at $532 \mathrm{~nm}$ (solid red line) and $1064 \mathrm{~nm}$ (solid blue line). The dashed lines correspond to the $n_{2, \text { eff }}$ fitting, taking into account the uncertainties.
$1.064 \mu \mathrm{m}$ [15], in contrast to $n_{4}=-2 \times 10^{-35} \mathrm{~m}^{4} / \mathrm{W}^{2}$ at

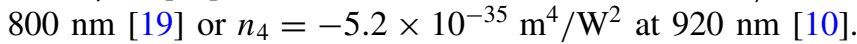
The change in the sign of $n_{4}$ with wavelength is related to the location of the third harmonic with respect to the absorption bands of the material. The main point is that, in the present situation, the saturation of the Kerr effect cannot be accounted for by the $n_{4}$ term. One may consider the option of retaining more terms in the expansion of the response function of the material in a power series of the electric field (higher-order nonlinear susceptibilities). However, two difficulties would arise then. First, from theoretical point of view, the expansion cannot remain valid when the higher-order corrections get the same order of magnitude as the leading one. In [10] it was possible to have a $n_{4}$ term comparable to the $n_{2}$ one because the third-harmonic resonance increased considerably the former with respect to the latter, which was not resonant. At the wavelengths considered in this paper, no such resonance effect is observed. Second, there is a practical difficulty in the measurement: Accurate values of $n_{2}$ are difficult to obtain; the difficulty is considerably increased for $n_{4}$, etc., so the accuracy required to reproduce the experimental results in our computations would not be reached with enough confidence.

One may think that nonlocality plays a role in these variations. The time response of the component of $n_{2, \text { eff }}$ due to molecular reorientation in $\mathrm{CS}_{2}$ was measured a long time ago [23] and was found to be about $1.6 \mathrm{ps}$. Hence it is less than the pulse duration for one order of magnitude, so retardation effects are small and will have no consequence at all on spatial behavior. Spatial nonlocality has been studied and has been found to have appreciable effects if the distance covered by excited atoms due to diffusion is appreciable with respect to the beam radius [24]. In contrast to gases, this distance is very small in liquids, especially for times in the picosecond range. Hence nonlocality is completely negligible here.

Hence we provide an empirical model that describes the variations of $n_{2}$ with intensity we observe experimentally by fitting the experimental curve with an adequate Ansatz. After several trials, including the expressions $n_{2, \mathrm{eff}}(I)=a I \exp \left(-b I^{c}\right)$ and $n_{2, \mathrm{eff}}(I)=a I /\left(1+b^{2} I\right)^{c}$, with $c=1$ or 2 , and $n_{2, \text { eff }}(I)=a I /\left(1+b^{2} I^{2}\right)^{2}$, we find that the Ansatz

$$
n_{2, \mathrm{eff}}(I)=\frac{a I}{1+b^{2} I^{2}}
$$

allows us to correctly reproduce the data at 532 and $1064 \mathrm{~nm}$. To adjust the parameters $a$ and $b$ in (5), we use the Levenberg-Marquardt nonlinear fit algorithm [25,26], as already implemented in the GNU OCTAVE software. The best fit, for data at $532 \mathrm{~nm}$, is obtained with the set of parameters $a=3.3921 \times 10^{-32} \mathrm{~m}^{4} / \mathrm{W}^{2}$ and $b=5.7643 \times 10^{-15} \mathrm{~m}^{2} / \mathrm{W}$, while at $1064 \mathrm{~nm}, \quad a=6.2989 \times 10^{-33} \mathrm{~m}^{4} / \mathrm{W}^{2}$ and $b=2.2673 \times 10^{-15} \mathrm{~m}^{2} / \mathrm{W}, \quad$ as illustrated in Fig. 5.

The NLS equation, in its most general form, reads

$$
i \frac{\partial E}{\partial z}=-\frac{1}{2 n_{0} k} \Delta_{\perp} E-F(|E|) E,
$$

in which $\Delta_{\perp}=\partial^{2} / \partial x^{2}+\partial^{2} / \partial y^{2}$ is the transverse Laplacian operator accounting for the paraxial diffraction, $k$ is the wave 
vector in vacuum, $n_{0}$ is the linear refractive index, and $F(|E|)$ is some function, real in the conservative case, that accounts for the nonlinearity. In the cubic case (pure Kerr effect),

$$
F(|E|)=\frac{3 k}{2 n_{0}} \operatorname{Re}\left(\chi^{(3)}\right)|E|^{2} .
$$

If absorption is taken into account up to fifth order, as fifth-order susceptibility, it becomes

$$
F(|E|)=k^{2}\left[i \operatorname{Im}\left(\chi^{(1)}\right)+3 \chi^{(3)}|E|^{2}+10 \chi^{(5)}|E|^{4}\right] .
$$

Here $\chi^{(1)}, \chi^{(3)}$, and $\chi^{(5)}$ are the optical susceptibilities of the first, third, and fifth order, respectively, which are complex numbers. The real part of $\chi^{(1)}$ does not appear in Eq. (6); it is incorporated into the index $n_{0}$. Note that the NLS equation including damping is then formally identical to the cubicquintic Ginzburg-Landau (CGL) equation. However, the CGL equation is always considered in the presence of gain while, if only dissipation is present as in the present situation, the properties are fundamentally different and essentially driven by those of the conservative case.

The evolution of the phase $\varphi$ is given by Eq. (2), in which, as we have seen previously, the effective nonlinear refractive index $n_{2 \text {,eff }}(I)=n_{2}+n_{4} I$ must be replaced with the experimentally observed variation law, according to Eq. (5). The intensity I follows Eq. (1), in which, for sake of simplicity and taking into account the results of Ref. [15], the coefficients $\alpha$ and $\beta$ can be set to zero, which yields

$$
\begin{gathered}
\frac{\partial \varphi}{\partial z}=\frac{k a I}{1+b^{2} I^{2}} I, \\
\frac{\partial I}{\partial z}=-\gamma I^{3} .
\end{gathered}
$$

We intend to define the function $F(|E|)$ to be used in the NLS equation (6) in accordance with Eqs. (9) and (10). We apply the thin sample approximation to Eq. (6), i.e., the diffraction term involving $\Delta_{\perp} E$ is set to zero. The amplitude and the phase are separated by substituting $|E| \exp (i \varphi)$ for $E$ in Eq. (6). Then the imaginary part yields, after simplification, the evolution equation of the phase $\varphi$,

$$
\frac{\partial \varphi}{\partial z}=\operatorname{Re}[F(|E|)]
$$

The real part is multiplied by the conjugate $E^{*}$ of $E$ to yield, after substitution of $|E|^{2}=I / 2 c \epsilon_{0} n_{0}$, the equation for the intensity $I$,

$$
\frac{\partial I}{\partial z}=-2 \operatorname{Im}[F(|E|)] I .
$$

Comparing Eqs. (11) and (12) with Eqs. (9) and (10) allows us to identify both the real and imaginary parts of $F(|E|)$. Substituting this function into the NLS equation (6) yields the model equation

$$
i \frac{\partial E}{\partial z}=-\frac{1}{2 n_{0} k} \Delta_{\perp} E-\left[\frac{k a I^{2}}{1+b^{2} I^{2}}+i \frac{\gamma I^{2}}{2}\right] E .
$$

For the numerical solution it is more convenient to work with the normalized form of the equation

$$
\frac{\partial u}{\partial z}=\frac{i}{2} \Delta u+i \frac{\eta|u|^{4} u}{1+|u|^{4}}-\mu|u|^{4} u,
$$

where $\Delta$ is the Laplacian operator relative to normalized space variables $X=x / w_{0}, Y=y / w_{0}, Z=z / l$, and $u=$ $E / E_{r}$, with $l=n_{0} k w_{0}^{2}$ and $E_{r}=1 / \sqrt{b 2 n_{0} c \epsilon_{0}}$ being the reference length and electric field, respectively. The field is normalized with respect to the intensity $1 / b$ at which $n_{2, \text { eff }}$ reaches its maximum value. Further, $\eta=l k a / b^{2}$ and $\mu=\gamma l / 2 b^{2}$.

Equation (14) is solved numerically by means of a standard fourth-order Runge-Kutta scheme in $z$ using five-point finite differences for the computation of the transverse Laplacian operator and absorbing boundary conditions. The D4 $\sigma$ method is used on the image at the entrance of the cell to get an accurate measurement of the beam diameter: $2 w_{0}=34 \mu \mathrm{m}$. Computations have been run over a propagation distance

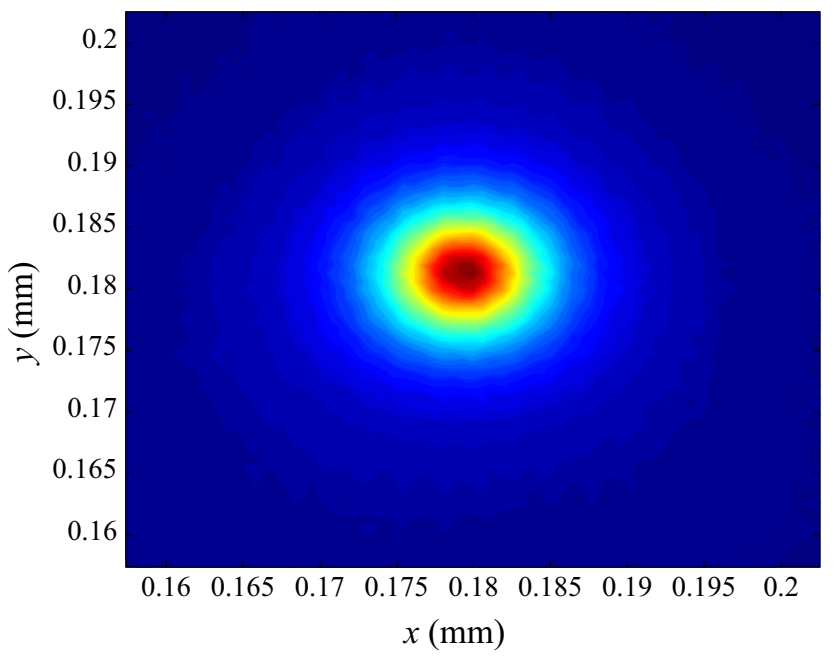

(a)

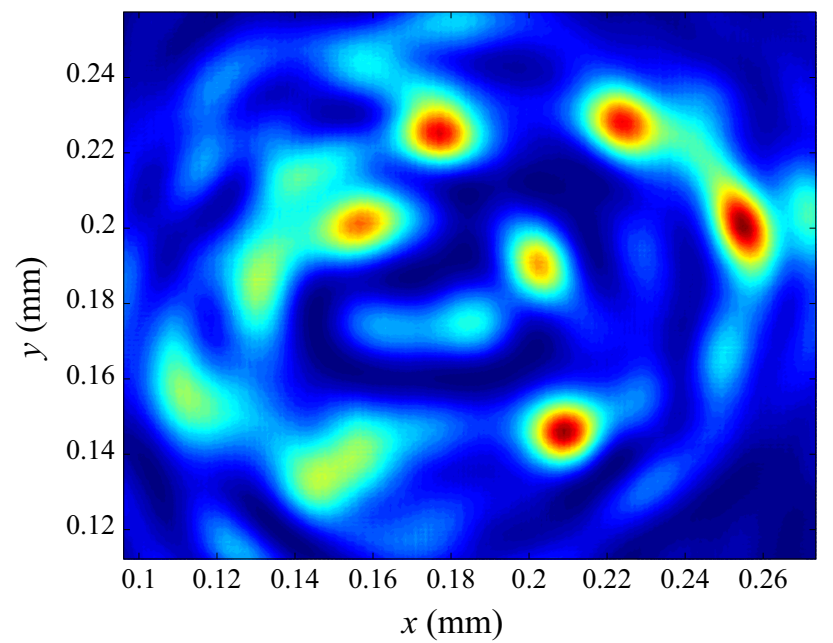

(b)

FIG. 6. (Color online) Intensity pattern after 10-mm propagation according to Eq. (13) for the input intensity (a) $I_{0}=5.54 \mathrm{GW} / \mathrm{cm}^{2}$ and (b) $I_{0}=139 \mathrm{GW} / \mathrm{cm}^{2}$. 
$z_{\max }=10 \mathrm{~mm}$, corresponding to the cell thickness. The normalized propagation distance is $Z=0.14$, the reference intensity is $I_{r}=17.348 \mathrm{GW} / \mathrm{cm}^{2}$, and the reference length is $l=70.369 \mathrm{~mm}$. We used a spatial step size $d X=d Y=$ $1.057 \times 10^{-2}$ and the normalized nonlinear parameters are $\eta=848.45$ and $\mu=98.479$.

The input data used for calculations is an image of the beam at the entrance of the cell recorded by the camera. This profile is multiplied by an adequate constant, so its maximum value becomes $|E|=\sqrt{I_{0} / 2 n_{0} c \epsilon_{0}}$, where $I_{0}$ is the required peak intensity. The background noise of the camera was subtracted numerically from the input signal. To avoid excessive computation time and data storage, the width of the computation domain is restricted to $3 w_{0}$.

For moderate intensities $\left(1-5.53 \mathrm{GW} / \mathrm{cm}^{2}\right)$ diffraction occurs. Then a first filament is formed for $I_{0}=5.54 \mathrm{GW} / \mathrm{cm}^{2}$ (see Fig. 6). A second filament appears when the input peak intensity is $I_{0}=15 \mathrm{GW} / \mathrm{cm}^{2}$.
It is seen from the profiles that the shape of the filaments is close to a Gaussian distribution. The numerical results are found in good agreement with experimental ones.

\section{CONCLUSION}

Accurate measurements of the nonlinear optical parameters of $\mathrm{CS}_{2}$ have shown unexpected variations of the effective nonlinear index $n_{2, \text { eff }}$ with intensity, which call into question the use of this liquid as a reference material for $n_{2}$ measurements. Further, the saturation of the Kerr effect, which is responsible for the formation of filaments, cannot be using the standard $n_{2}$ -

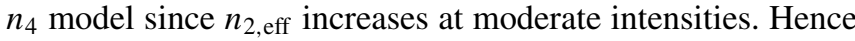
an adequate model has been built to reproduce the formation of filaments observed in experiments. Further research is planned with the aim of controlling the filaments location by manipulating the input beam phase and shape, for example, by means of a spatial light modulator.
[1] V. I. Bespalov and V. I. Talanov, Pis'ma Zh. Eksp. Teor. Fiz. 3, 471 (1966).

[2] A. Couairon and A. Mysyrowicz, Phys. Rep. 441, 47 (2007).

[3] G. N. Steinberg, Phys. Rev. A 4, 1182 (1971).

[4] O. G. Kosareva, T. Nguyen, N. A. Panov, W. Liu, A. Saliminia, V. P. Kandidov, N. Akozbek, M. Scalora, R. Vallee, and S. L. Chin, Opt. Commun. 267, 511 (2006).

[5] L. Bergé, S. Skupin, F. Lederer, G. Méjean, J. Yu, J. Kasparian, E. Salmon, J. P. Wolf, M. Rodriguez, L. Wöste, R. Bourayou, and R. Sauerbrey, Phys. Rev. Lett. 92, 225002 (2004).

[6] G. Fibich and B. Ilan, Opt. Lett. 26, 840 (2001).

[7] A. Dubietis, G. Tamosauskas, G. Fibich, and B. Ilan, Opt. Lett. 29, 1126 (2004).

[8] H. Gao, X. Sun, B. Zeng, S. Xu, W. Chu, W. Liu, Y. Cheng, Z. Xu, and G. Mu, J. Opt. 14, 065203 (2012).

[9] A. Sukhinin and A. B. Aceves, J. Opt. 15, 044010 (2013).

[10] E. L. Falcão-Filho, C. B. de Araújo, G. Boudebs, H. Leblond, and V. Skarka, Phys. Rev. Lett. 110, 013901 (2013).

[11] M. Sheik-Bahae, A. A. Said, T. H. Wei, D. Hagan, and E. W. Van Stryland, IEEE J. Quantum Electron. 26, 760 (1990).

[12] D. Milam, Appl. Opt. 37, 546 (1998).

[13] R. A. Ganeev, A. I. Ryasnyansky, M. Baba, M. Suzuki, N. Ishizawa, M. Turu, S. Sakakibara, and H. Kuroda, Appl. Phys. B 78, 433 (2004).
[14] R. A. Ganeev, A. I. Ryasnyanski, and H. Kuroda, Opt. Spectrosc. 100, 108 (2006).

[15] V. Besse, G. Boudebs, and H. Leblond, Appl. Phys. B (to be published).

[16] G. Boudebs, S. Cherukulappurath, M. Guignard, J. Troles, F. Smektala, and F. Sanchez, Opt. Commun. 232, 417 (2004).

[17] S. Cherukulappurath, J. L. Godet, and G. Boudebs, J. Nonlinear Opt. Phys. Mater. 14, 49 (2005).

[18] G. Boudebs, S. Cherukulappurath, H. Leblond, J. Troles, F. Smektala, and F. Sanchez, Opt. Commun. 219, 427 (2003).

[19] D. G. Kong, Q. Chang, H. A. Ye, Y. C. Gao, Y. X. Wang, X. R. Zhang, K. Yang, W. Z. Wu, and Y. L. Song, J. Phys. B 42, 065401 (2009).

[20] G. Boudebs and K. Fedus, J. Appl. Phys. 105, 103106 (2009).

[21] G. Boudebs, V. Besse, C. Cassagne, H. Leblond, and C. B. de Araújo, Opt. Lett. 38, 2206 (2013).

[22] V. Skarka, V. I. Berezhiani, and R. Miklaszewski, Phys. Rev. E 56, 1080 (1997).

[23] D. McMorrow, W. T. Lotshaw, and G. A. Kenney-Wallace, IEEE J. Quantum Electron. 24, 443 (1988).

[24] D. Suter and T. Blasberg, Phys. Rev. A 48, 4583 (1993).

[25] K. Levenberg, Q. Appl. Math. 2, 164 (1944).

[26] D. Marquardt, SIAM J. Appl. Math. 11, 431 (1963). 